\title{
INTRACELLULAR NEURONAL RECORDING WITH FLEXIBLE MICRO-MACHINED PROBE IMPLANTS
}

\author{
Anupama V. Govindarajan ${ }^{1}$, Tai C. Chen ${ }^{1}$, Russell C. Wyeth ${ }^{2}$, \\ A. O. Dennis Willows ${ }^{2}$ and Karl F. Böhringer ${ }^{1}$ \\ ${ }^{1}$ Department of Electrical Engineering, ${ }^{2}$ Department of Zoology, University of Washington \\ Seattle Washington 98195
}

\begin{abstract}
We present an implantable sub-micron silicon probe device, with flexible interconnects, that is capable of intracellular neuronal recording. The silicon probe device's ability to penetrate and record from neurons in an isolated brain of the sea slug Tritonia diomedea was tested, and we demonstrate that, for the first time, an implantable device can record intracellular action potentials from a single neuron (Pd5 of the Tritonia brain). Our group has focused on intracellular neuronal recording with stand-alone silicon needles in the past. In this paper we present the integration of the needle, using standard microfabrication techniques, with a flexible interconnect to an aluminum base pad where electrical connections to the device can be made. Intracellular signals provide detailed information about the functioning of single neurons by directly tracking the transmembrane voltage, as opposed to only recording the field potentials caused by large changes in the transmembrane voltage as is the case in extra-cellular recordings. We have found that the silicon probes (needles) have lower impedances than the $\mathrm{Ag} / \mathrm{AgCl}$ glass capillary electrodes used conventionally for similar intracellular recording purposes. The success of this prototype is a major step toward self-contained, implantable devices suited for intracellular neuronal recording from freely behaving animals.
\end{abstract}

\section{INTRODUCTION}

MEMS devices are particularly promising as neuronal recording tools due to their small dimensions, compact geometry, and the ease with which multi electrode recording and integration with on-chip electronics can be performed. Recent research has successfully demonstrated electrical microprobes made from silicon that are suitable for neuronal recording [1]. These silicon microprobes with typical cross-sections of tens of micrometers can penetrate living tissue without causing significant trauma $[1,2]$.

Many MEMS devices for extra-cellular neuronal recording have been presented [1-5]. However, to answer many interesting neurobiological questions, intracellular neuronal recordings are needed. Neurons process inputs and produce outputs through modulation of the transmembrane voltage. By directly measuring the DC state of the cell, intracellular recordings can provide detailed information about the functioning of single neurons and their synaptic interactions. In contrast, extracellular recordings, at best give only the timing of action potentials fired by a single neuron - a far more limited view of the neuron's activity.

Standard techniques for intracellular recording consist of glass capillaries and fine conducting wires. Pulled glass capillaries or micropipettes can be transformed into intracellular neuronal probes by filling them with an electrolyte and placing an $\mathrm{Ag} / \mathrm{AgCl}$ electrode in the electrolyte. The glass walls provide ionic insulation and ensure low leakage current, while the tapered tip produced by pulling the capillary is small enough to penetrate neurons without damaging them. However these micropipettes have overall large dimensions and limited ability to integrate with components such as memory, amplifiers and power sources in a compact manner that will allow measurements from freely behaving animals [6]. Previous MEMS based approaches for intracellular neuronal recording using silicon probes used standalone needles, which are not suitable for implantation. [7].

In this paper we outline the significant changes to the process flow described in [6-8] that have enabled the integration of the high aspect ratio, sub-micron silicon probes to a larger silicon base bearing an aluminum pad for electrical connections (Fig. 1). The interconnecting scheme uses two polyimide layers to sandwich a metallic aluminum layer (Pi-Al-Pi interconnect). The metal layer forms the electrical connection between the $\mathrm{Al}$ pad on the large silicon base and the silicon needle, while the polyimide layers provide the flexible insulation. This new device was tested on neurons in an isolated brain of the sea slug Tritonia diomedea. We have demonstrated that intracellular action potentials from a single neuron (Pd5 of the Tritonia brain) can be recorded by the silicon needle device, connected via the $\mathrm{Al}$ pad to a conventional neurobiological amplifier. Our success is a major step toward the larger goal of self-contained, implantable devices suited for intracellular neuronal recording from freely behaving animals (Fig. 2).

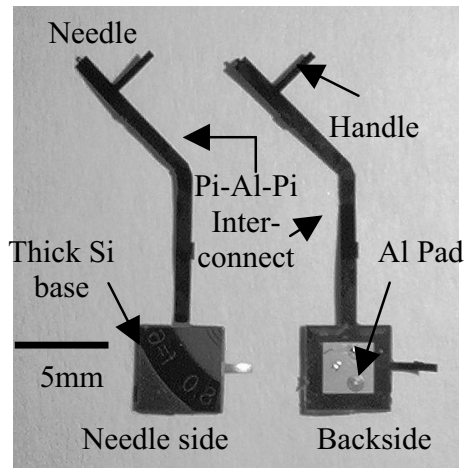

Figure 1. Completed submicron silicon probe devices produced by a combination of micromachining and precision laser cutting.

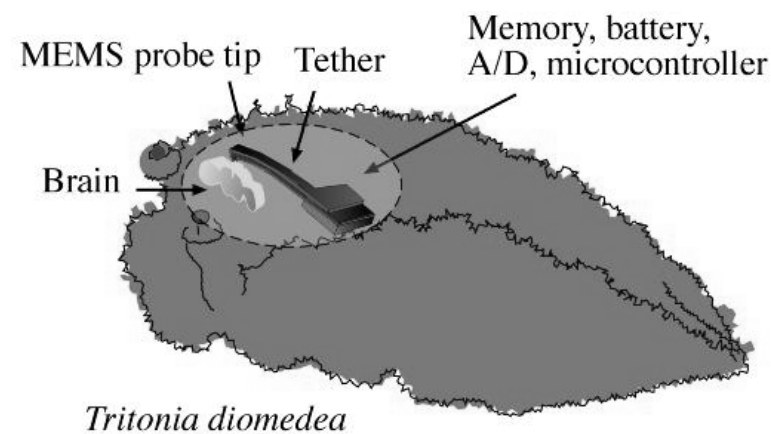

Figure 2. Schematic of Tritonia diomedea with envisioned standalone neural recording implant [8]. Animal length is $10 \mathrm{~cm}$, neurons can be as large as $400 \mu \mathrm{m}$. Brain dimensions are several $\mathrm{mm}$. (Integrated electronics not included in current needle design.)

Travel support has generously been provided by the Transducers Research Foundation and the DARPA MEMS and DARPA BioFlips programs. 


\section{FABRICATION}

We started with a $400 \mu \mathrm{m}$ thick, 4", <100> DSP boron doped silicon wafer with a resistivity of $0.1-0.6 \Omega$-cm. First a $1000 \AA$ aluminum etch-stop layer was patterned (Fig. 3b). This protects the polyimide interconnect during the needle sharpening in $\mathrm{SF}_{6}$ plasma. Next the wafer was backside aligned and patterned with photoresist AZP4620 (Clariant), to define the needle, release trench and large silicon base using the standard Bosch process in the Deep-Reactive-Ion-Etching (DRIE) system (Fig. 3c). The needle probes $(385 \mu \mathrm{m}$ tall) had protective rings around them to ensure positive profiles while sharpening. Removal of DRIE residue required treatment with $\mathrm{CF}_{4} \mathrm{O}_{2}$ plasma in addition to EKC 830 resist strip.

The wafer was flipped over and the Pi-Al-Pi interconnect was defined (Fig. 3d-f). The second layer of polyimide was cured at a higher temperature than the first layer. This approach terminates any route for water transmission through the boundary between the base and top polyimide layers [9], ensuring a sound electrical connection between the needle and the Al pad. This curing approach also anneals the $\mathrm{Al}$, ensures better adhesion and a reduced contact resistance.

Before sharpening, the Pi-Al-Pi side was covered with a layer of resist, to prevent the etching of the polyimide in $\mathrm{SF}_{6}$ plasma from the under side of the wafer, as well as to protect the $\mathrm{Al}$ electrical connection layer when the $\mathrm{Al}$ etch stop layer is etched away following the sharpening. Needles were simultaneously sharpened (Fig. 3g) by Reactive-Ion-Etching (RIE) using $\mathrm{SF}_{6}$ plasma. During the sharpening the protective ring is etched away. Fig. 4 shows a sharpened silicon needle with a sub $\mu \mathrm{m}$ tip inset. The silicon at the base of the trench is also etched away during the sharpening, exposing the $\mathrm{Al}$ etch stop layer.

At this point the device is attached to the wafer only at the polyimide tabs and in the trench surrounding the thick silicon base. The Al etch stop layer was etched away (Fig. 3h) by carefully immersing the wafer in $\mathrm{Al}$ etchant (etch rate $~ 3500 \AA / \mathrm{min}$ at $40^{\circ} \mathrm{C}$ ) keeping the wafer in vertical position so as to prevent the weight of the etchant from stressing the needle devices. After this the protective backside resist was stripped. The wafer was finally immersed in Isopropyl Alcohol (IPA), which cleaned the wafer before it evaporated leaving a dry surface. At this point we have high aspect ratio, sub micrometer silicon needles connected to a silicon base through a Pi-Al-Pi sandwich. The individual devices are finally released by laser ablation, in which the polyimide tabs that hold the device to the wafer are cut.

\section{RESULTS AND DISCUSSION}

The resistance between the silicon probe (needle) and a ground wire, both immersed in sea water, was $1 \mathrm{M} \Omega$, on the application of a $100 \mathrm{~Hz}+/-10 \mathrm{nA}$ square wave current generated by A-M Systems neuroprobe 1600 amplifier in combination with a stimulator (Medical systems Corp. Model S-90). The resistance of traditional $\mathrm{Ag} / \mathrm{AgCl}$ glass capillary electrodes is $5-60 \mathrm{M} \Omega$. The rough contour of the needle leads to increased surface area for the given needle geometry. This increased surface area might contribute to lowering the impedance of the needle.

We used the sea slug Tritonia diomedea as a model system to measure the intracellular action potentials for a variety of reasons, the foremost being that the brain of this animal has been extensively studied and the extremely large neurons in its brain make for very easy conventional recordings. To measure the action potentials, the brain of Tritonia was isolated in a dish containing seawater along with a ground wire. Using scissors, a dorsal

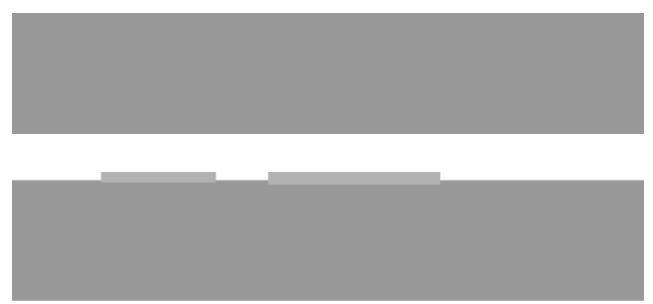

(a)

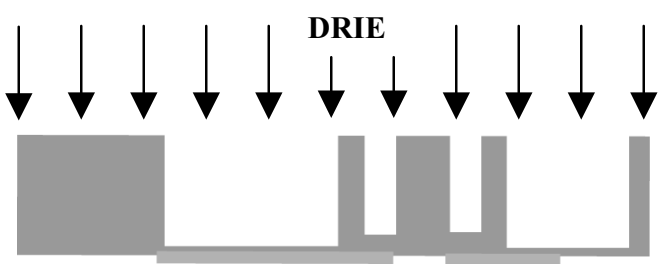

(c)

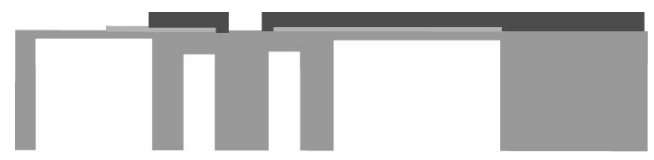

(b)

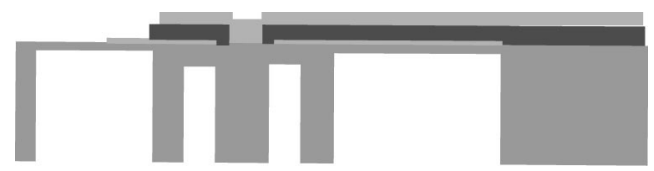

(e)

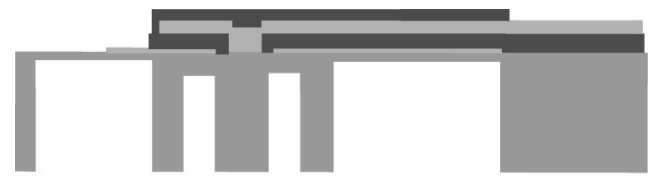

(d)

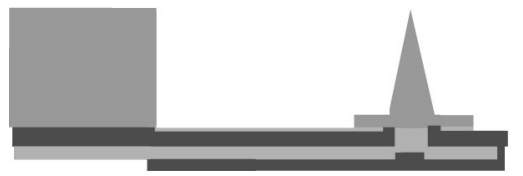

(g)

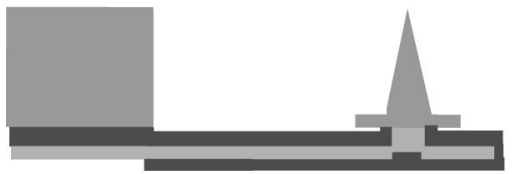

(h)

Initial Needle $\varnothing=70 \mu \mathrm{m}$, Ring thickness $=40 \mu \mathrm{m}$

$\mathrm{Gap}=50 \mu \mathrm{m}$ (to ensure positive profile while sharpening).
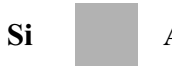

Al

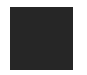

Polyimide

Figure 3. Fabrication of the sub-micron silicon probe device; (a) Bare Si wafer, (b) Al etch stop layer patterning on wafer backside, (c) Defining Si probes with DRIE on needle side, (d) Polyimide layer 1 patterning and curing on wafer backside, (e) Al electrical contact layer patterning, (f) Polyimide layer 2 patterning and curing, (g) Needle front side sharpening with RIE, (h) Al etch stop layer etch. Individual devices released by laser cutting. 


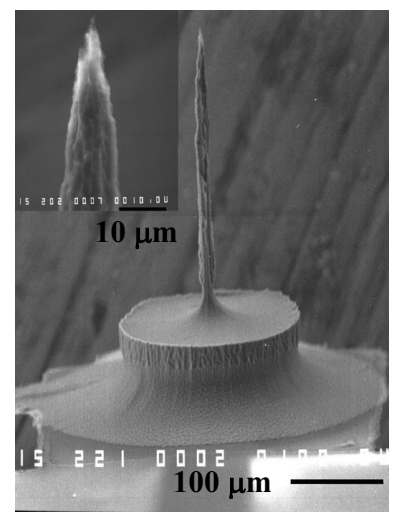

Figure 4. SEM images of the submicron silicon probe with tip inset.

Needle height is $\sim 400 \mu \mathrm{m}$.

Needle tip diameter is far less than $1 \mu \mathrm{m}$.

Needle: Magnification 221x, scale bar $100 \mu \mathrm{m}$

Tip: Magnification 2000x, scale bar $10 \mu \mathrm{m}$.

incision was made overtop of the brain. All nerves emanating from the brain were cut, along with the associated connective tissue, allowing its removal from the body of the animal. The isolated brain was then pinned in a dish, stretched taut and any remaining connective tissue was dissected away. This configuration promotes conventional micropipette penetration of brain neurons, and we reasoned would do the same for silicon needle recording. Figure 5 shows a picture of an isolated Tritonia brain.

We measured the voltage between the needle inserted in the Pd5 neuron and the ground wire immersed in the seawater surrounding the isolated brain, using an A-M Systems Neuroprobe 1600 Amplifier. The headstage of the amplifier was connected to the needle probe via the Al pad on the large silicon base. The voltage signal was digitized using a Cambridge Electronic Design Micro1401 MkII with Spike 2 Version 4 software (Fig. 6). As the needle was not insulated, a non-conductive layer of mineral oil was dispensed over the brain to record only from the probe tip because the needle pushed through the oil into the neuron. The oil layer molded to the surface of the brain, creating a high resistance path between the probe tip and the ground wire, reducing the short to the ground enough to allow us to record neuronal signals.

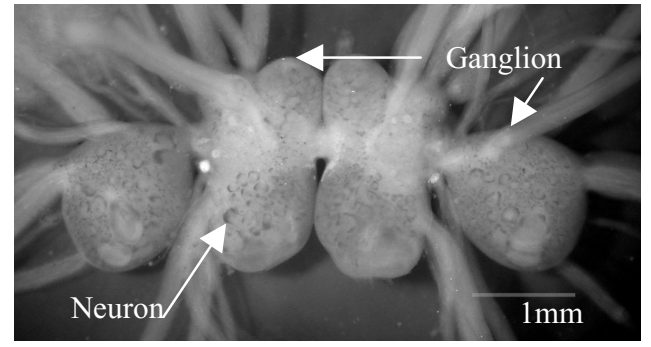

Figure 5 . Isolated brain of Tritonia diomedea, showing the ganglia and neurons.

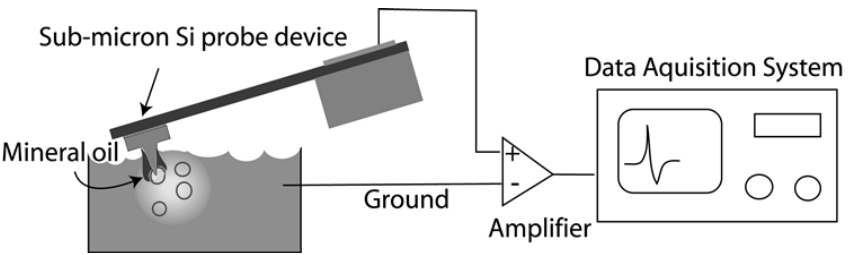

Figure 6. Schematic of the experimental and electronic setup. (Mineral oil layer is shown only on the neuron of interest for clarity).

For penetration experiments, the needle tip was manipulated by a tapered glass capillary, which was glued to the back side of the needle base using cyanoacrylate glue (Fig. 7). The non tapered end of the capillary was attached to a micromanipulator. Similarly, the large silicon base of the needle device containing the Al base pad connected to the amplifier headstage was held in another micromanipulator. Both the silicon needle device and the tapered capillary were manipulated into position over a target neuron, before a penetration attempt was made. Fig. 8 shows the needle on top of the $\mathrm{Pd} 5$ neuron just before penetration. Penetration comprises slowly advancing the needle along its tip-to-base axis, interspersed with very gentle taps of the manipulator - an identical method to penetration using conventional micropipettes. The flexible interconnect arm allows a range of independent movement of the needle, facilitating final manipulations during penetration. In this fashion we were able to penetrate single neurons and measure intracellular transmembrane potentials with an implantable device.

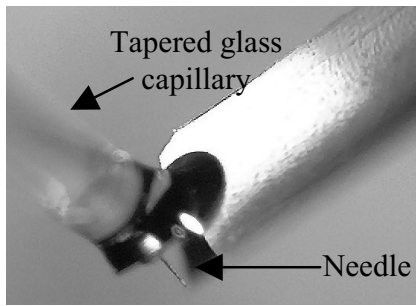

Figure 7. Preparation for cell penetration. (silicon probe is manipulated by tapered glass capillary attached by veterinary glue).

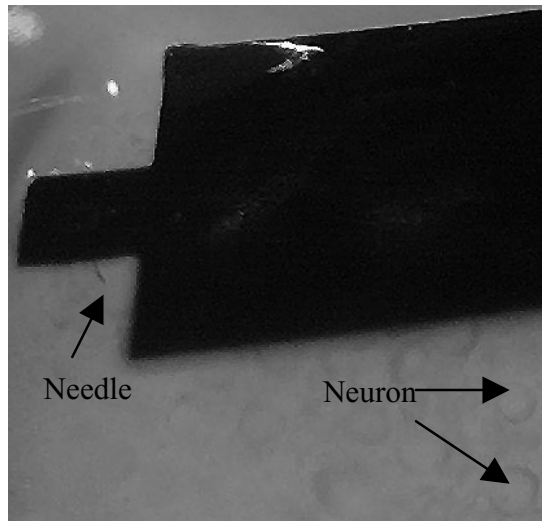

Figure

8. Implantation of $a$ sub-micron silicon probe into a specific neuron - Pd5 of the brain of Tritonia diomedea.

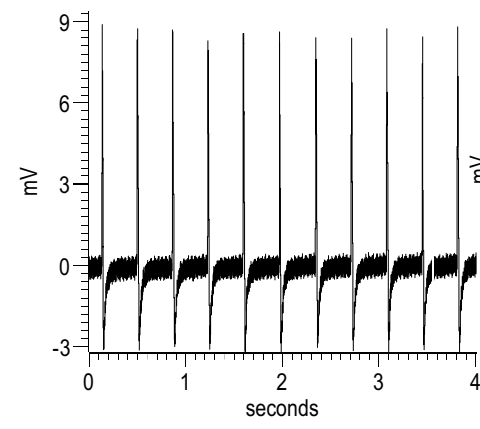

(a)

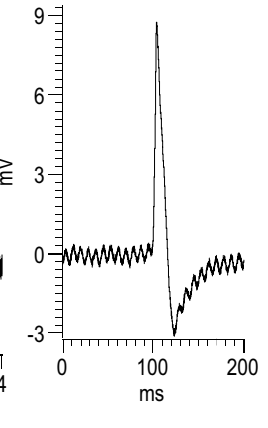

(b)
Figure 9. Intracellular action potentials recorded from Pd5 neuron of the Tritonia brain using a sub-micron silicon probe; (a) Train of action potentials, (b) Single action potential with its three phases: a fast initial depolarization, a fast repolarization, followed by a slow hyperpolarization before returning to the baseline.

Fig. 9 shows the intracellular action potentials recorded from the Pd5 neuron of the Tritonia brain with the implantable silicon probe device. The $12 \mathrm{mV}$ potentials have the form of attenuated, low frequency filtered action potentials recorded using conventional glass electrodes. In particular, they show all three major phases of an action potential: a fast initial depolarization, a fast repolarization, followed by a slow hyperpolarization before 
returning to the baseline. Circumstantial evidence supports the hypothesis that these action potentials are intracellular:

1) The spike shapes look like intracellular, particularly after the hyperpolarization, which is quite distinctive. 2) The spike sizes, though small, are in the intracellular range. Extracellular action potentials would be in the range of $\mu \mathrm{V}$. Further, the amplitude of the recording did not increase as we advanced the probe, rather the recording started with a discrete change from no activity to full potentials, consistent with sudden intracellular penetration.

\section{FUTURE WORK}

It is tempting to speculate that the imperfect insulation provided by the oil allowed some short to the ground, reducing the size of the action potentials. While using glass micropipettes the cell membrane attaches itself to the sides of the glass electrode thus sealing the neuron from the external environment. With this in mind the next step in our process would be needle insulation with $\mathrm{SiO}_{2}$. The major challenge here is the deposition of $\mathrm{SiO}_{2}$ on the polyimide as well as the needle.

Implantation of the silicon probe device into the brain of a freely moving organism would require efforts to ensure that the probe does not move relative to the neuron it has penetrated. After penetration the base of the needle needs to be glued to the perineurium - the acellular layer that bounds the brain in sea slugs. This will create a low profile bump on the brain, which we hope will not be susceptible to movement of the brain inside the animal. For this, the needle will have to be manipulated with a detachable device rather than the glued and tapered glass capillary. Changing the geometry of the polyimide arm to a spiral shape will increase the flexibility of the connection between the needle and the large silicon base, so that when they move relative to one another there will be reduced stress on the needle.

We would also like to extend the capability of our device to perform neurostimulation by coating the tip of the needle with metals of high exchange current density [10].

\section{SUMMARY}

In conclusion, we have fabricated implantable silicon probe devices with flexible interconnects suitable for intracellular neuronal recording. These probes were implanted into a specific neuron (Pd5) of the Tritonia brain and for the first time we have shown that an implantable device can successfully measure intracellular action potentials. The silicon probes have reduced impedances compared to the $\mathrm{Ag} / \mathrm{AgCl}$ glass capillary electrodes used conventionally for intracellular neuronal recording. These probes can be easily integrated with electronic circuitry to make them self-contained.

\section{ACKNOWLEDGEMENT}

The authors would like to thank Dr. Giles Dennler (Linz Institute for Plastic Solar Cells, Johannes Kepler University of Linz, Linz, Austria) for detailed discussions about the behavior of PECVD deposited $\mathrm{SiO}_{2}$ on Polyimide.

This research was supported by David and Lucile Packard Foundation grant 2000-01763.

\section{REFERENCES}

1. L. Lin and A. P. Pisano, "Silicon-Processed Microneedles", IEEE Journal of Microelectromechanical Systems, vol. 1, no. 1, March 1999.
2. K. Najafi, K. D. Wise and T. Mochizuki, "A High-Yield ICCompatible Multichannel Recording Array", IEEE Transactions on Electron Devices, vol.ED-32, (1985), pp.1206-1211.

3. D. J. Anderson, K. Najafi, S. J. Tanghe, D. A. Evans, K. L. Levy, J. F. Hetke, X. Xue, J. J. Zappia, and K. D. Wise, "Batch Fabricated Thin-Film Electrodes for Stimulation of the Central Auditory System", IEEE Transactions on Biomedical Engineering, vol. 36, (1989), pp. 693-704.

4. G. T. A Kovacs, C. W. Storment, M. Halks-Miller, C. R. Jr. Belczynski, C. C. D. Santina, E. R. Lewis and N. I. Maluf, "Silicon-Substrate Microelectrode Arrays for Parallel Recording of Neural Activity in Peripheral and Cranial Nerves", IEEE Transactions on Biomedical Engineering, vol. 41, (1994), pp. 567577.

5. P. K. Campbell, K. E. Jones, R. J. Huber, K. W. Horch and R. A. Normann, "A Silicon Based, Three Dimensional Neural Interface: Manufacturing Processes for an Intracortical Electrode Array", IEEE Transactions on Biomedical Engineering, vol. 38, (1991), pp. 758-768.

6. Y. Hanein, C. G. J. Schabmueller, G. Holman, P. Luecke, D. D. Denton, K. F. Böhringer, "High-Aspect Ratio Submicrometer Needles for Intracellular Applications", IOP Journal of Micromechanics and Microengineering (JMM) 13(4):S91-S95, July 2003.

7. Y. Hanein, U. Lang, J. Theobald, R. Wyeth, K.F. Böhringer, T. Daniel, D.D. Denton, and A.O.D. Willows, "Intracellular Recording with High Aspect Ratio MEMS Neuronal Probes", Proceedings of the International Conference on Solid-State Sensors and Actuators (Transducers), (2001), Munich, Germany.

8. G. Holman, Y. Hanein, R. C. Wyeth, A. O. D. Willows, D. D. Denton, K. F. Böhringer, "Silicon Micro-Needles with Flexible Interconnections", Second International IEEE-EMBS Special Topic Conference on Microtechnologies in Medicine \& Biology, pp. 255-260, May 2-4, 2002, Madison, Wisconsin.

9. K. Lee, A. Singh, H. Zhu, G. Coryell, B. Olsen, B. kim, G. Raupp and J. He, "Fabrication of Implantable Polyimide Based Neural Implants with Flexible Regions to Accommodate Micromovement", Twelfth International Conference on Solid State Sensors, Actuators and Microsystems, vol. 2, June 8-12 2003, Boston, pp. 1221-1224.

10. G. T. A. Kovacs, "Introduction to the Theory, Design and Modeling of Thin-Film Microelectrodes for Neural Interfaces", chapter in, Enabling Technologies for Cultured Neural Networks, D. A. Stenger and T. McKenna, Eds., Academic Press, 1994, pp. 121-166.

11. S. Takeuchi, T. Suzuki, K. Mabuchi and H. Fujita, "3D Flexible Multichannel Probe Array", IEEE The Sixteenth Annual International Conference on Micro Electro Mechanical Systems, Jan 19-23 2003, Kyoto, Japan, pp. 367-370. 